\title{
En quête de l'essentiel : la poésie dénudée chez Henri Bosco
}

\author{
Covadonga GRIJALBA CASTAÑOS \\ Universidad de Almería \\ grijalba@ual.es
}

Recibido: 23/10/2011

Aceptado: 20/04/2012

Résumé

Une approche de l'œuvre poétique d'Henri Bosco, la moins connue de l'auteur, à travers un recueil de compositions brèves, Des nuages, des voix, des songes... nous dévoile une sorte de poésie dénudée, libre d'artifices dans sa thématique et dans son style, d'une apparente simplicité, qui nous transporte du monde visible et tangible de la nature qui l'entoure, vers l'intérieur de l'âme. C'est de là qu'émergent les sentiments les plus intimes, les rêves et les mystères incarnés dans l'imaginaire profond du moi du poète, résumé de la sagesse accumulée au cours de sa longue et prolifique existence, et dévoilés dans ces poèmes composés un an avant sa disparition.

Mots clés: Henri Bosco, poèmes courts, poésie dénudée.

\section{En busca de lo esencial: la poesía desnuda de Henri Bosco}

\begin{abstract}
Resumen
Una aproximación a la obra poética de Henri Bosco, la menos conocida de este autor, a través de un volumen de composiciones breves, Des nuages des voix, des songes... nos desvela una poesía desnuda, liberada de artificio en su temática y estilo, de una sencillez aparente, que nos transporta del mundo visible y tangible de la naturaleza que le rodea, hacia el interior del alma. Es de allí de donde afloran los más íntimos sentimientos, sueños y misterios, inmersos en el imaginario profundo del yo del poeta, resumen de la sabiduría acumulada en su larga y prolífica vida, y desvelados en estos poemas compuestos un año antes de su desaparición.
\end{abstract}

Palabras clave: Henri Bosco, poemas breves, poesía desnuda.

\section{In search of the essential: the naked poetry of Henri Bosco}

\begin{abstract}
An approach to Henri Bosco's poetry - the least known of this author's genres - through a volume of short compositions, Des Voix des Nuages, des songes ... reveals a naked poetry, free of thematic artifice and apparently marked by stylistic simplicity. His poetry transports us from the tangible nature surrounding the author into his soul. Deeply immersed in the imaginary ego of the poet, the most intimate feelings, dreams, and mysteries emerge from the soul, like an inventory of the accumulated wisdom in his long and prolific life, divulged in these poems written a year before his passing.
\end{abstract}

Keywords: Henri Bosco, short poem, naked poetry. 


\section{Referencia normalizada}

Grijalba Castaños, C. (2013). "En quête de l'essentiel : la poésie dénudée chez Henri Bosco". Thélème, Vol. 28, 157-170.

Dans la deuxième décennie du XXe siècle, la vie littéraire française est animée par des controverses autour du concept de poésie pure. Il s'agit d'une idée originale de Mallarmé, mais dénommée, volontairement ou involontairement, par Paul Valéry, et qui constitue en elle-même une définition, ainsi que la naissance d'un problème quand on l'assimile à la pensée ${ }^{1}$. Des courants d'adeptes et de détracteurs se sont affrontés entre 1925 et 1930, nourris par le discours de l'abbé Brémond à l'Académie française La poésie pure, le 24 octobre 1925 ; sa thèse se résume en quelques mots : ce qui est véritablement poétique, c'est l'ineffable, l'inexprimable, l'indicible; paroles très proches de ce qu'avait exprimé avant lui Valéry : ce que l'on peut communiquer en prose n'est pas digne de faire partie d'un poème. C'està-dire que l'on invite à séparer l'essence poétique de toute autre matière qui lui soit étrangère.

Plusieurs spécialistes ont fourni des définitions à propos du sens général de ce terme en le mettant en correspondance avec l'existence de la musique pure, la raison pure ou la morale pure. «La poésie devient pure quand, au sein d'un poème, on fait abstraction de tout élément non expressément poétique» (Tverdota, 1996 : 301). Voici une autre définition qui s'oriente vers les mêmes paramètres :

Poesía pura es todo lo que permanece en el poema después de haber eliminado todo lo que no es poesía. Pura es igual a simple químicamente. Lo cual implica pues una definición esencial (Diego, 1932 : 197, cité par Azam, $1986: 145)$.

Cette allusion à la chimie évoque l'alchimie lyrique qu'on situe à la base de la poésie pure, pour exprimer le principe de l'élimination ou de l'épuration, appliqué à extraire l'or de la boue, et dont nous parle Tverdota (1996:308). Car, si la poésie pure comporte l'abstraction de tout élément non expressément poétique, elle rejoint la mystique et le rationalisme, marquant ainsi la coupure entre les mystiques de l'âme, représentés para les poètes romantiques, et les mystiques de la technique poétique.

Chez nous, la poésie de Juan Ramón Jiménez a été classée comme poésie pure, une attribution que le poète préfère éviter pour accueillir celle de poésie dénudée; ce nouveau terme est le fruit d'un parcours par les étapes de la contemplation, la

${ }^{1}$ La thèse de Valéry sur la poésie à l'état pur s'appuie sur Edgar Allan Poë et sur la mélomanie de Baudelaire, la situant à la croisée de la poésie et la musique. Les premiers débats ont eu comme origine sa Préface « Connaissance de la Déesse » figurant dans la plaquette de Lucien Fabre, en 1920. 
pénétration et le dévoilement, appliquées à un autre poète, Jorge Guillén (Azam, 1986 : 148). C'est-à-dire que dans la création, le poète est capable de pénétrer la réalité et d'établir le lien entre celle-ci et son être, comme résultat d'un processus de connaissance profonde qu'il réinterprète, recrée et réélabore à la lumière d'une interminable révélation de l'individualité créatrice.

Poesía desnuda sería el desvelamiento sucesivo en un yo sucesivo que vive al día la eternidad [...] una nueva visión del mundo que centra su interés en lo desnudo, en lo eterno subyacente a lo aparente, concreto y tangencial (Sotelo, $1991: 87$ ).

L'explication incorpore implicitement une certaine identification de la poésie et de l'âme, ainsi qu'une épuration formelle de l'idée. D'après les paroles précédentes c'est avec de nouveaux yeux que le poète contemple la réalité ; des yeux capables d'en extraire l'essentiel et où finalement, dans sa quête de ce genre de poésie, il retrouve les sphères les plus intimes de son identité d'homme. Et pour atteindre ces domaines poétiques il doit parcourir un long chemin jusqu'à la trouvaille du mot précis, capable de présenter la plénitude des choses et des êtres tout en conservant uniquement l'essentiel du langage, l'expression de l'âme, la pensée et les sentiments simples et purs.

Mais laissons la poésie pure au milieu des disputes et concentrons-nous sur les poèmes courts d'Henri Bosco. La froideur des théories esthétiques n'ajoutera peutêtre rien à la poétique conçue comme expression des sentiments d'un esprit sensible qui s'avère aussi un maître des mots, qui les étudie, les contourne, les épure, les situe là où ils doivent être placés, et pour lequel, même avec les limitations matérielles concernant les mots, ils constituent le seul moyen d'exprimer le noyau de sa pensée.

Pour une approche de ces poèmes de Bosco, nous avons recours à l'expression poésie dénudée, qui indique un dépouillement des points de vue formel et thématique, en réponse à une esthétique et une éthique de l'essentiel et de la simplicité.

Des images, des voix, des songes... est le titre d'un bref recueil de 27 petits poèmes d'Henri Bosco (1888-1976), une œuvre simple et profonde qui traduit l'esprit et l'âme de son auteur. Les textes, édités en 1980 et illustrés par Liliane Marco, avaient été composés en $1975^{2}$, une année avant la mort de l'auteur. L'ensemble constitue un intéressant document littéraire-pictural, qui nous met en contact avec un univers très cher au poète.

Bien connu par son œuvre en prose, Bosco, que ses parents envisageaient d'orienter vers la musique, grâce à ses études au Conservatoire d'Avignon, avait tout enfant découvert la poésie, à travers les histoires que son père lui racontait pour l'aider à s'endormir, capables d'éveiller sa curiosité et de se constituer en source

\footnotetext{
${ }^{2}$ Chaque poème écrit sur un bristol et manuscrit a été envoyé par l'auteur à la destinataire de la dédicace, sa collaboratrice Monique Baréa, qui nous en a fait la confidence.
} 
inépuisable d'inspiration pour son œuvre postérieure. La curiosité et les rêves qui peuplaient ses premières années en milieu familial d'abord, et que ses maîtres se sont chargés de nourrir à l'école ensuite, vont donner naissance à sa vocation littéraire.

C'est un cor, le cor d'Obéron, qui m'a révélé, tout enfant, la poésie. [...] Tout étant naturellement incroyable, il n'y avait rien qui ne répondit au désir caché que j'avais en moi du féerique, du surnaturel, de l'impossible (Bosco, 1966 : 175-176).

Et cette attirance trouve sa continuité au Lycée : «Dans ces noires bâtisses ils [ses professeurs] nous enseignaient la lumière » (Bosco, 1984a : 7-8), surtout avec Aristide Brun, «Inoubliable maître à qui je dois d'avoir lu Théocrite et Virgile comme s'ils avaient parlé provençal » (Bosco, 1987: 47), « il ne vivait qu'entre le Parnasse et l'Olympe, et nous avec lui » (Bosco, $1958: 120$ ), et qui lui fait connaître la poésie du monde classique gréco-latin et la poésie bucolique; grâce à ce maître, il apprécie un proche voisin, l'Homère provençal, le poète Mistral. Avec de telles influences il ne pouvait plus qu'exprimer: «Tel fut le meilleur, le plus inoubliable des hommes qui illumina mon adolescence [...] C'est lui qui a décidé ma vocation littéraire. Je lui dois beaucoup du peu que je suis » (Bosco, 1984b : 30-31).

Il a 13 ans quand la revue suisse La Renaissance lui accorde la Médaille d'or pour son poème Étoile de la mer, inspiré d'une chanson liturgique. Ses premières compositions étaient de longues séries de vers de claire inspiration virgilienne ${ }^{3}:$ « Je suis virgilien dans l'âme. Virgile, je crois, c'est le poète auquel, toutes proportions gardées, je ressemblerais le plus » (Cauvin, 1974 : 252). Il compose vers 1906 la Trilogie de l'Espoir ou Poèmes de l'Espoir, repris comme Les poètes, aux éditions des Terrasses de Lourmarin. Lors de ses études supérieures à Florence il se familiarise avec les poètes italiens du dolce stil nuovo et il s'apprête à imiter Dante en écrivant une Divine Comédie française que finalement il détruira. Et à côté des Italiens, il découvre les poètes et les philosophes grecs, les mystiques espagnols et germaniques, et une vaste palette parmi les Français : les romantiques Lamartine, Hugo ou Musset; La Bruyère ${ }^{4}$; La Fontaine, qui le séduit par sa manière de faire parler les animaux; Racine, Maurice de Guérin et Nerval. Évidemment il ne pouvait pas oublier les grands poètes provençaux, Mistral et Joseph d'Arbaud, représentants du caractère méditerranéen, dont il admirait le sens profond, le «merveilleux provençal, mi-païen, mi-chrétien » (Cauvin, 1974: 30). Des poètes du XXe

\footnotetext{
${ }^{3}$ II avait longtemps travaillé cet auteur et traduit ses Bucoliques; il écrira ses propres Bucoliques de Provence, publiées dans la collection « Les Relais de Fontaine », Alger-Tunis, Éditions de la Revue Fontaine -créée par Max-Pol Fouchet- 1944. Ces poèmes ont été repris dans Le Roseau et la Source.

${ }^{4}$ Qu'il imite au Lycée pour composer des poèmes-portraits de ses collègues et de ses professeurs ; petites compositions à la rime simple qu'il lit, relit et polit minutieusement, " comme s'ils étaitent des objets taillés dans un bois dur» ("Lettre inédite à M.E. Coindreau, le 6 janvier 1946 » in Cauvin, $1974: 37)$.
} 
siècle, il respectait Valéry et Supervielle à qui il dédiera plusieurs poèmes dans $L e$ Roseau et la Source.

Tout au long de sa vie, Bosco a donc fait preuve d'évolution poétique, et est passé du style pseudoclassique et même baroque d'inspiration philosophique, à la poésie enfantine, les chansons traditionnelles, les poèmes spirituels et mystiques, et finalement, le poème court, lucide et vivace.

Certes il abandonnera cette voie poétique pour entreprendre sa carrière littéraire en prose, mais il ne cessera jamais d'écrire des poèmes dont il parsème les pages de romans, récits, souvenirs, etc., et qui sont restés inédits ou bien ont été publiés dans diverses revues et recueils; des poèmes élégiaques ou de signe religieux, sortes de prières et de chansons populaires en français ou dans sa langue maternelle le provençal, dont il composait aussi la musique. Toute son œuvre majeure n'a été qu'une transposition de la poésie dans la prose, exemple d'un parfait mariage des deux formes d'expression littéraire, ce qui eut comme conséquence des difficultés de classification auxquelles l'auteur contribua :

Le mot poésie veut dire création. Et on peut être poète dans un roman [...] Mais [...] un livre comme Malicroix, ou dans un livre comme L'Âne Culotte, ce sont des véritables poèmes pour moi (Ytier, $1966: 7)^{5}$.

D'autre part, il considère le récit comme un moyen d'atteindre la poésie, à laquelle il attribue, dans son sens le plus ample, la fonction d'instrument de connaissance de l'âme humaine, d'une authentique quête intérieure:

C'est la poésie que je cherche, c'est-à-dire la création de fictions tirées du fond de l'âme et dont la vie fictive analysée avec soin me permet d'étudier et de connaître cette âme elle-même. (Lettre de H. Bosco en Steinmann, 1963: 216).

Comme nous le rappelle María Zambrano, qui a étudié les relations entre la philosophie et la poésie: «La poesía es un abrirse del ser hacia dentro y hacia fuera al mismo tiempo, es un oír en el silencio y un ver en la obscuridad «la música callada, la soledad sonora » (Zambrano, $2001: 110)$.

Toute la pensée poétique bosquienne se concentre dans son credo recueilli dans «L'Exaltation et l'amplitude» (Bosco, 1942), où il nous avertit de ses propos : «La poésie n'est pas un jeu, mais un moyen de haute connaisance » (Bosco, 1942 : 273). Il parle du poème comme d'une créature qui essaie d'imiter Dieu dans son action créatrice, et du poète comme d'un créateur qui redonne la vie en évoquant les formes, les images, les figures mentales, les pensées, les sentiments, le corps, la matière, tout le " créé », car il ressuscite les créatures et « puis, sans les voir, et par pure divination il appellera les plus belles figures » (Bosco, $1942: 276)$.

\footnotetext{
${ }^{5}$ Malicroix sera classé comme roman, conte épique ou poème, et Bachelard considère même cette œuvre comme « un vaste poème en prose » (Bachelard, 1992 : 56).
} 
Ces réflexions nous réaffirment dans notre première attribution aux poèmes courts de Bosco du nom de poésie dénudée, concentrée sur l'essentiel de la langue pour traduire une âme et aspirer à l'essentiel de l'esprit.

\section{Approche thématique}

Les sujets abordés dans ce recueil reflètent les constantes de son imaginaire poétique, un univers personnel qu'il n'a jamais abandonné : la nature proche et simple, objet de son amour enthousiaste et source de bienfaits, capable de s'harmoniser avec l'homme ; la terre peuplée de fleurs, de forêts, d'eau, d'animaux, et couverte par l'espace immense de la voûte céleste, de préférence au moment de la nuit - qu'il craint et qu'il aime en même temps-; le monde des Ombres qui la traversent et enveloppent le mystère de la mort et le secret de la vie ; l'enfance naïve... des sujets universels qu'il reprend maintes fois dans son œuvre en prose.

Car à un moment donné Bosco avait fait une découverte non sans conséquences: qu'il avait reçu de la nature un don, celui de communiquer intimement avec les choses, les objets et les éléments de la nature. Ce don lui confère une sorte de sensibilité spéciale pour dévoiler la vie qui habite au fond des êtres, même les plus simples et inanimés. Il l'a utilisé comme source d'inspiration pour s'introduire dans la vie invisible et secrète où règne le double étrange, l'autre caché, son compagnon de songes, les présences inquiétantes, les âmes, les ombres, les rêves, le sens du mystère et même un monde dans son innocence première, un retour à l'initiale harmonie impossible à recouvrer, d'un paradis perdu : « La matière parle à sa façon. Si l'on est doué pour l'entendre, on l'écoute et parfois on la comprend » (Bosco, 1978: 301). Cette relation homme-nature est chez Bosco d'une identification pleine : «C'est pourquoi entre nous et les éléments naturels un merveilleux contact s'établit aussitôt. L'eau, la terre, le feu et l'air nous furent révélés » (Bosco, 1990 : 67)

La nature est exaltée, comprise, assimilée, justifiée dans ses êtres simples et petits qu'il aimait tant : les oiseaux, les grillons, la pluie, le vent, l'ombre, la neige, les bribes d'herbes, etc., et en compagnie desquels il se sentait à l'aise, en amicale communion: "Une incessante conversation va s'établir entre lui et le monde. L'Univers entier s'anime pour participer à la vie du personnage » (Descheaux, $1982: 205)$.

Les mots d'introduction d'un autre recueil, Le Roseau et la Source, soulignent cette participation : "Le ROSEAU chante/Et la SOURCE pleure/et moi j'écoute », comme évidence du rôle qu'un homme sensible accorde à la nature qu'il a appris à écouter attentivement. Cet incipit nous renvoie au proverbe touareg: "La flûte de roseau ne chante jamais si bien que dans la solitude où le silence écoute... », applicable à tant de roseaux des œuvres de Bosco; silence et solitude qui s'entremêlent et deviennent vraiment indispensables pour laisser se développer les songes et pour écouter les voix qui parlent de l'intérieur de l'homme, de n'importe quel homme, et 
bien sûr du poète : " Il [le poète] ne pensera plus par syllogismes, il pensera par songes » (Bosco, 1981b : 17).

Mais sa dévotion pour la nature et le sentiment qu'il en fait partie ne lui font pas oublier que l'homme, même attiré par les forces obscures de la terre, sait la dépasser pour rejoindre un ordre supérieur, celui de l'esprit. L'enfant qui disait «J'ai souvent écouté la terre » (Bosco, 1978:4) a toujours conservé cette attirance et cette sensibilité, que le poète cherche au sein de la réalité même et qu'il oriente vers le Jardin du Paradis : « Je ne savais pas que j'avais recueilli en moi assez de jardins, d'aube et de nuits, d'odeurs, de saveurs et aussi des visages —ah! surtout ces visages d'Ombre !» (Bosco, $1966: 169)$.

Une analyse concrète du recueil laisse entrevoir son éventail thématique :

Le poète, en observateur infatigable, décrit l'écoulement du temps qui s'écoule entre l'hiver et le printemps $(13)^{6}$ et qu'il reprendra encore entre ces deux saisons : hiver-printemps, jour et nuit (15), enfance, jeunesse ou vieillesse, et qu'il chante finalement pour exalter la fidélité aux principes ou aux premières amours (22). On constate que l'été et l'automne n'ont aucune place dans ces vers.

Il y a deux exemples de vie animale : un seul oiseau pour marquer les saisons (13) et une colombe qui clôt le dernier, symbole de la paix et de l'âme en pureté :

\author{
Offre une colombe \\ Au sommeil \\ Pour qu'une colombe \\ Entende tes songes (27).
}

Par contre, il y a de nombreuses références au monde végétal, aux ombres et aux rêves, car tout semble parler dans l'espace des songes, au milieu du sommeil ou en pleine veille. Le chêne et les arbres de la forêt se parlent dans l'ombre de la nuit (4) et d'autres plantes : le roseau (5), le lys (7), la rose (10 et 15), l'amandier (23), et le symbole de la vie par excellence, l'arbre mort qui fleurit et qui nous invite à la renaissance (25).

Trois des quatre éléments se donnent rendez-vous la nuit : le végétal (le roseau), le vent et l'eau représentée par la fontaine (5); des voix de la nature qui parlent, comme les arbres, au moment du crépuscule. Et le vent est repris dans l'ensemble des Quatre Vents du monde (10), voire dans tous les vents de la Rose, des vents importants, au nom propre.

L'eau forme un cercle qui part du ciel et revient aux nuages et inaugure le cycle de sa vie sur la terre (6). On trouve d'autres sortes d'eaux : de la mer (7), du lac (20), l'eau tranquille comme un miroir pour se regarder et se reconnaître (19), et la Source qui jaillit de la terre, qui provoque un respect sacral envers cette pureté (26) et qui laisse l'homme au seuil d'un mystère.

\footnotetext{
${ }^{6}$ Nous avons numéroté les poèmes pour en faciliter l'analyse.
} 
Dans le premier poème, à travers la musique, le poète nous situe au centre du monde ; il s'identifie à un instrument, le luth ${ }^{7}$ : ce n'est pas un instrument quelconque mais un luth aux Cordes d'Or; son appartenance à la sphère du Soleil marque les diférences avec un brin de majesté et de surnaturel dans ce chant où il invite le monde :

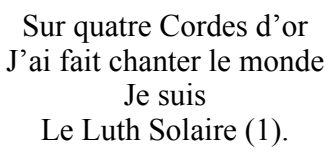

Et c'est dans le cosmos que les quatre points cardinaux recevront les prières (2). La terre, le ciel, les planètes, l'étoile (3 et 11), reprise explicitement (15) et implicitement (20). Et voilà une étoile spéciale qui n'a pas de nom, désignée comme la Seule (3) -le plus long poème du recueil—, soit l'Unique : douée d'un caractère extraordinaire, c'est peut-être celle qui conduisit les mages de l'Épiphanie, «l'astre des Rois » (Michel, $1991: 250$ ).

Chez un écrivain d'un pays constamment ensoleillé, on est surpris par l'attrait de l'obscurité de la nuit. Mais c'est dans ces conditions que les mystères se manifestent : «Il faut avoir fait amitié avec la nuit pour maintenir un sentiment si plein de la présence de la terre » (Bosco, 1981a : 9).

La présence de la nuit qui s'approche et la force de la nature ambivalente, dans la tempête (7), une nuit qui fait briller la lumière sur les eaux du lac (20) en illuminant la scène et en laissant une place pour que l'on voie vivre la lampe :

\author{
Sans la nuit \\ Que serait ta lampe? \\ Et sans toi \\ Que serait la nuit? (21)
}

Les ombres (4) créent l'ambiance la plus propice pour laisser la nature s'exprimer elle-même, et en particulier l'Ombre, avec majuscule, représentant l'âme (9) ou l'esprit (18). L'ombre en contraste avec la lumière est une image obsédante profondément imprégnée de mysticisme; l'ombre douée de pouvoirs lucides et dont l'absence ramène à l'isolement et à la solitude.

Et entre corps et âme (8), l'âme toujours présente (11), l'âme identifiée à un rêve

\footnotetext{
${ }^{7}$ Le luth, instrument de l'ancienne Mésopotamie répandu à travers l'Asie grâce à la Route de la Soie et en Europe à travers l'Espagne Islamique. Au IX siècle un musicien d'origine perse, Zyriab, créa les premières écoles dans la péninsule Ibérique. Il modifia le luth traditionnel en lui ajoutant une cinquième corde, de couleur rouge, qui représentait le pont entre la musique et l'âme. Même si l'instrument de Bosco n'avait que quatre cordes, la musique de ses poèmes nous renvoie à cette cinquième comme le pont qui relie musique et sensibilité de l'âme.
} 


\author{
Il dort \\ L'âme dans ses bras \\ Tous deux \\ Ne font qu'une même âme (24)
}

Ou à une force vivifiante (25). Les âmes enfermées dans les arbres. ${ }^{8}$ L'aspect spirituel se montre dans les âmes ainsi que dans les prières qu'il inscrit sur les points cardinaux (2) et la prière-songe pour bâtir des temples (16).

Précédant les points de suspension du titre, le mot songes nous introduit dans le monde onirique bosquien, car les songes « avaient ouvert la patrie secrète dans laquelle j'allais entrer pour en faire ma vie » (Bosco, 1961 : 307). Une fois installés là il n'est pas facile d'établir les limites, «À force de former des rêves la vie réelle pouvait elle-même devenir à son tour un rêve » (Bosco, 1966 : 260). C'est dans ce monde onirique que l'on peut trouver la clé pour comprendre le retour de Bosco aux domaines de l'enfance : «le seul et véritable âge du rêve, c'est celui de l'enfance » (Bosco, 1966 : 153), le paradis. Et les rêves ou les songes (16 et 17), en communion âme-rêves (24), songes et sommeil (27).

Bosco introduit le personnage du double, un double obscur qu'il avait traité magistralement dans Un Rameau de la Nuit. Et grâce au don reçu il peut communiquer avec lui. Le poème qui parle du double nous fait évoquer le titre d'un de ses récits : Mon compagnon de songes, mais à qui s'adresse-t-il ici? (12) à son double, à l'autre, en tout cas dans ce poème, à un compagnon de songes (14).

La présence enfantine (23) innocente et naïve, fait évoquer le paradis perdu.

La quête du paradis perdu dont nous portons en nous le regret plus encore que le souvenir, et ce regret se fait créateur, il engendre des fictions qui projettent paradoxalement dans le futur, l'image de l'âge d'or.

Une telle quête passe donc par le rêve et l'enfance retrouvée, mais elle attire l'orage et les périls. (Girault, $1984: 38$ ).

Il y a dans ces poèmes des enfants, d'habitude très jeunes, livrés aux rêves et aux jeux propres de l'âge dont l'évocation dans ses Souvenirs produit chez l'auteur une certaine nostalgie:

Cet enfant $[\ldots]$ c'est lui dont je cherche dans ma vieillesse à ramener à moi les peines, les douleurs, les joies, les plaisirs, les pensées et surtout les songes, car c'est toujours par eux que je les reconnais, s'il est vrai, comme je le sens, que nous n'avons fait à nous deux, qu'un seul et même songe, depuis notre apparition sur la terre.

Et que serais-je sans ce songe ?... (Bosco, 1981c : 5-6).

\footnotetext{
${ }^{8}$ Sujet familier à Bosco qui l'a traité dans Le Jardin d'Hyacinthe et surtout dans Mon Compagnon de Songes. Sujet classique aussi que déjà Dante représentait, dans le Chant XIII de l'Enfer de sa Divine Comédie avec les âmes enfermées à l'intérieur des troncs d'arbres.
} 
Les sujets récurrents : la nature, le cosmos, la musique, l'âme, la spiritualité, l'homme, les rêves, les songes, l'ombre, l'enfant, l'état de veille ou d'endormissement, convergent à la lumière de la nuit multipliée en des myriades de petites étincelles. Quand le poète contemple la voûte céleste, le poème parle du soleil, des étoiles, des planètes, mais il s'éloigne d'un sujet très cultivé en particulier par les poètes romantiques qu'il admirait tant, un sujet qui a rempli des milliers de pages depuis l'aube de la poésie : la lune; il va sans dire qu'elle devenait très utile dans ses récits en temps de difficultés, au milieu des obscures ténèbres réelles ou imaginées.

La diversité de motifs pour un recueil si bref met en relief la cohérence d'un univers harmonieux à moitié suspendu dans le temps ; le lecteur se sent attiré par la beauté des illustrations, des images et des mots de ce paysage intérieur incarné en paroles dépouillées ; une beauté faite d'un savoir ancien et d'une quête en solitaire. Ancrés dans des paysages définis, ces poèmes s'inscrivent sous le signe d'une intimité pleine d'images de l'essentiel d'une vie, qui parle de la terre avec bonheur ou avec un respect mystérieux et qui nous invite à construire des ponts entre l'ici et l'ailleurs.

Des images, des voix, des songes... nous semblent devoir s'appréhender comme des morceaux détachés d'une expérience spirituelle, des moments forts d'un cheminement créateur qui serait à retracer (Michel, $1992: 248$ ).

En somme, ce recueil est une invitation à renouer le contact avec la vie simple et vibrante de la nature, à l'aimer tendrement et à se mettre en harmonie avec elle ; et Bosco, tel un troubadour provençal qui chantait l'amour courtois, se présente comme un Provençal qui chante l'amour de la nature.

\section{Approche poétique}

La musique des vers nous transporte à travers un monde naturel d'apparence simple, et des mots dépouillés de tous leurs accessoires: une simplicité qui exprime l'essence d'une pensée longtemps cultivée, la réflexion qui invite le lecteur à l'approfondir et à ne pas se satisfaire d'une première impression, pour essayer d'en dégager le noyau essentiel et primitif, générateur d'extrême concision, et qui se montre comme l'unique voie pour atteindre la connaissance.

Ces compositions sont intemporelles, même si elles évoquent un passé accompli qui s'actualise, comme une constatation des évidences d'une réalité qui nous semble proche, quoiqu'elle soit voisine du rêve. Poèmes autonomes qui dessinent le parcours d'une autobiographie poétique aux jalons spatiotemporels.

Poésie simpliste ? Certainement pas! L'apparente simplicité provient du recours aux mots les plus simples mais situés à l'endroit et au moment précis du poème, un ici et là qui vise un au-delà. Un lexique bien connu n'empêche pas l'originale et surprenante combinaison qu'éveillent chez le lecteur des échos d'une expérience vécue, proche et lointaine à la fois, sous de nouveaux registres. Et le dialogue qui s'était 
établi entre le poète et les mots répète le dialogue préalable entre le poète et la nature ou les êtres ; une certaine communion met en rapport tous les éléments de la création, dans une fraternité tout à fait naturelle, comme nous le montre Zambrano:

Y el milagro de la poseía surge en plenitud cuando en sus instantes de gracia ha encontrado las cosas, las cosas en su peculiaridad y en su virginidad, sobre este fondo último; las cosas renacidas desde su raíz. [...] Ya el hombre es sólo voz que canta y manifiesta el ser de las cosas y de todo. [...] La palabra que define y la palabra que penetra lentamente en la noche de lo inexpresable [...] porque no se resigna a que cada ser sea solamente lo que aparece (Zambrano, 2001 : 114-115).

Mais voilà que ce naturel renferme des mystères, des ombres, des paroles, des faits, qui dépassent le naturel proprement dit. Car, comme on peut lire, « No hay buena poesía sin poesía » (Petan, 2002 : 62), et chez Bosco, «Être poéte, c'est d'abord obéir à la poésie » (Fouchet, 1979 : 183). Tous ces éléments semblent agir en force génératrice qui s'impose au poète, qui arrive lorsqu'elle le veut et non lorsqu'on la réclame : «La parole vient à la bouche, l'histoire commence... Il n'y a plus qu'à l'écouter, et, en ce moment je l'écoute» (Bosco, 1990 : 9), nous informet-il à propos de son processus créateur.

Poésie hermétique ? Seulement en apparence, car en poésie ce qui intéresse c'est surtout la vérité, et elle est ici embaumée de caractères qui la voilent ou la montrent un peu mystérieusement et capable par elle-même d'éveiller les émotions ; il s'agit d'une poésie somme toute rigoureuse et essentielle, marquée par la sobriété comme procédé et comme exercice de recherche de l'essentiel.

Le poids des vers libres repose sur les substantifs et se poursuit à travers des verbes au passé ou à l'impératif qui s'adressent non seulement au lecteur mais peutêtre aussi, au poète lui-même. Des mots d'une grande sobriété qui rapprochent ou qui éloignent en même temps ; parfois des énumérations de substantifs qui mettent en valeur les éléments d'une nature toujours protagoniste: le roseau, le vent, la fontaine (5) ou l'eau, la terre, la mer, les nuages, rapprochés dans une série causeeffet (6).

En l'absence de rime, libéré de cette discipline, le poète établit un jeu de rythmes changeants grâce aux différents mètres, leur principale caractéristique étant le goût de la parole et les idées sous-jacentes exprimées dans un lexique clair et direct, plein de sensibilité. Leur brièveté ne comporte aucune banalité ; ces poèmes, il les a distillés comme de petites gouttes de son esprit sensible et poétique, qu'il concrétise en très peu de mots et en très peu de lignes, disposées sur la page sous une forme aléatoire : ce sont des sortes de calligrammes, de nuages épars entre les ombres et les lumières des illustrations, formant un archipel ${ }^{9}$ graphique noir sur blanc dans le ciel de la page manuscrite originale, noir sur gris dans les lithographies de Liliane Marco, et où l'essentiel, peu évident, ne réside pas dans la forme et nous échappe au

\footnotetext{
${ }^{9}$ On pourrait appliquer ice le sens que René Char donne à cette expression et qui sera l'objet du titre de son recueil La parole en archipel (1962).
} 
premier abord : c'est cette idée qu'exprime la célèbre phrase du chapitre XXI du Petit Prince : «L'essentiel est invisible pour les yeux ».

Ce ne sont plus alors des mots couchés sur la page qui s'offrent à son regard [du lecteur], à son écoute, mais des mots en relief. Il les ressent gonflés d'une forme mystérieuse ; il entend en eux un chant informulable [...] deux ou trois mots en relief qui font exister chaque poème court de Henri Bosco $[\ldots]$ limpides $[\ldots]$ et sournoisement magiques et inquiétants $[\ldots]$ des mots ferments de ses récits (Michel, $1992: 247$ ).

L'exercice de lecture impose une vision en abyme pour déceler l'esprit des mots et l'âme du poème ; il s'établit une sorte de monologue intérieur que le poète avait déjà effectué avec les choses en s'interrogeant à propos de divers aspects de ce qu'il perçoit d'une façon sensible ou imaginaire, et révélatrice d'un esprit en totale harmonie avec son environnement.

Et à côté des images, survolant tous les poèmes, les voix, qui parlent de toutes les manières possibles, nous laissent entrevoir l'essence des choses qui demeurent secrètes, et auxquelles la parole prête une visibilité pour cacher d'autres évidences secrètes.

\author{
J'ai écouté \\ Il chantait. Qui était-ce? \\ Moi qui vous parle \\ Ou bien l'autre qui songe? (14)
}

Et encore le silence qui semble vibrer au fond de chaque poème soit implicitement soit suggéré par les points de suspension ou d'interrogation, des interrogations sans réponse aucune, lancées en l'air, qui agissent comme des ellipses des idées sous-jacentes, en laissant s'écouler une sorte de mystère. La réalité demeure somme toute à demi voilée et un peu inaccessible aux sens :

Les poèmes courts qui composent Des nuages, des voix, des songes... laissent la part la plus importante au Silence. Ils constituent l'essence d'une activité poétique inséparable d'une démarche spirituelle (Michel, $1992: 253$ ).

Les propos de Carriedo sur le silence, appliqué à Guillevic ${ }^{10}$, conviennent aussi à notre auteur: "'Silencio en continua búsqueda de sí mismo' que contribuye a configurar ese paisaje de la desnudez en exacta consonancia con el espacio interior » (Carriedo, $1992: 43)$.

Poésie assujettie à une grande rigueur et à un travail minutieux, résultat d'un processus de mûrissement accompagné d'une vie de silence et de réflexion sur l'homme, et sur l'essentiel de l'être. Le recueil est le reflet d'une expérience sur la

\footnotetext{
${ }^{10}$ Plusieurs réflexions dans l'article de Carriedo sur Guillevic pourraient s'appliquer à notre étude, étant données les similitudes conceptuelles et formelles entre les deux poètes.
} 
langue et sur la vie en quête d'une poésie épurée, pour dire l'intime d'une âme. Sans doute qu'il considérait la Poésie « comme exercice spirituel ${ }^{11}$.

De la lecture des textes de ce recueil se dégage un sentiment de légèreté évanescente, une impression de pur état de l'âme. Les mots, tels une paroi transparente, se laissent pénétrer et permettent de voir au-delà d'eux-mêmes ce qui était réservé : le vrai sens, le mystère du monde et de l'âme humaine ainsi que l'âme du poète qui ne meurt jamais et devient génératrice d'autres vies :

\author{
J'ai enfermé \\ cette âme \\ Dans un arbre. \\ On le croyait mort \\ Et il a fleuri. (25)
}

\title{
RÉFÉRENCES BIBLIOGRAPHIQUES
}

Azam, G., (1989) "Ser y estar en la poesía pura" in Neumeister, S. (dir.), Actas del IX Congreso de la Asociación Internacional de Hispanistas. Vol. 2, pp. 143-152. Bachelard, G., (1992) La Poétique de l'Espace. Paris, PUF.

Bosco, H., (1942) "L'exaltation et l'amplitude" in Fontaine. №19-20, Alger, pp. 273-276.

Bosco, H., (1944) Bucoliques de Provence. Alger/Tunis, Les Relais de Fontaine.

Bosco, H., (1949) Le Roseau et la Source. Paris, Gallimard.

Bosco, H., (1958) "Diaire inédit. Début mai" en Cahiers Henri Bosco. $\mathrm{N}^{\circ}$ 15, juin 1978.

Bosco, H., (1958) Pascalet. Paris, Gallimard.

Bosco, H., (1961) Un oubli moins profond. Paris, Gallimard.

Bosco, H., (1966) Le Jardin des Trinitaires. Paris, Gallimard.

Bosco, H., (1967) Mon Compagnon de Songes. Paris, Gallimard.

Bosco, H., (1978) Un rameau de la nuit. $1^{\text {ere }}$ édition 1950. Paris, Gallimard.

Bosco, H., (1978) Une Ombre. Paris, Gallimard.

Bosco, H., (1980) Des nuages, des voix, des songes... Illustrations de Liliane Marco. Aix-en-Provence, Edisud.

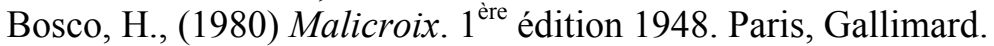

Bosco, H., (1981a) "Puissance de la terre dans Maurice de Guérin" in Cahiers Henri Bosco. No 21, pp. 9-14.

${ }^{11}$ Paroles qui constituent le titre de la Préface que Max-Pol Fouchet écrivit pour la revue Fontaine ( ${ }^{\circ}$ 19-20, mars-avril 1942), dédié aux relations entre poésie et mystique et où il fait la défense de la grande poésie, celle qui fait penser aux problèmes essentiels de l'homme et à la transcendance. C'est dans ce numéro spécial qu'apparaît l'article de Bosco « L'Exaltation et l'Amplitude ». 
Bosco, H., (1981b) "Henri Bosco par lui-même" en Cahiers Henri Bosco. №21, pp. 15-18.

Bosco, H., (1981c) "Souvenirs" in Cahiers Henri Bosco. No 21, pp. 5-6.

Bosco, H., (1983) L'Enfant et la rivière. $1^{\text {ere }}$ édition 1953. Paris, Gallimard.

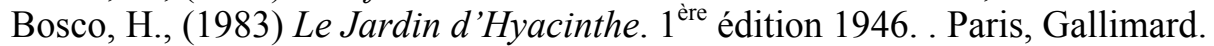

Bosco, H., (1984a) "Discours d'Avignon (Citoyenneté d'honneur), septembre 1970" in Cahiers Henri Bosco. N²4, pp. 6-8.

Bosco, H., (1984b) "Il y a cinquante ans..." in Cahiers Henri Bosco. №24, pp. 6-32.

Bosco, H., (1987) "Provence" in Cahiers Henri Bosco. No27, pp. 43-52.

Bosco, H., (1990) Le Renard dans l'île. $1^{\text {ère }}$ édition 1956. Paris, Gallimard.

Bosco, H., (1992) Antonin. $1^{\text {ère }}$ édition 1952. Paris, Gallimard.

Carriedo, L., (1992) "La poesía de la desnudez de Eugène Guillevic" in Revista de Filología Francesa. № 2, pp. 39-49.

Cauvin, J. P., (1974) Henri Bosco et la poétique du sacré. Paris, Klinksieck.

Char, R., (1962) La parole en archipel. Paris, Gallimard.

Descheaux, B., (1982) L'imagination des éléments et leur fonction dans l'œuvre romanesque d'Henri Bosco. Thèse de Doctorat. Université de Bordeaux III.

Diego, G. (ed.), (1932) Poesía española, Antología 1915-1931. Madrid, Signo.

Fouchet, M.-P., (1968) "La poésie comme exercice spirituel" in Fouchet, M.-P., Un jour, je m'en souviens. Paris, Mercure de France, pp. 199-204.

Girault, Cl., (1984) "Correspondance inédite. À des lecteurs connus et inconnus" in Cahiers Henri Bosco. N²4, pp. 37-40.

Michel, J., (1991) "Réflexions sur le poème court de Henri Bosco" in Cahiers Henri Bosco. No30/31, pp. 247-255.

Petan, Z., (2002) Aforismos. Vitoria, Basari.

Sotelo Vázquez, A., (1991) "Poesía desnuda y sucesiva (Nuevas notas sobre la influencia de Miguel de Unamuno en Juan Ramón Jiménez)" in Anales de Literatura Española. №7, pp. 183-194.

Steinmann, J., (1963) Littérature d'hier et d'aujourd'hui. Paris, Desclée de Brouwer.

Tverdota, G., (1996) "La poésie pure en France et en Hongrie" in Revue d'Études Françaises. $\mathrm{N}^{\mathrm{o}} 1$, pp. 301-309.

Ytier, R., (1966) Henri Bosco ou l'amour de la vie. Lyon, Ed. Aubanel.

Zambrano, M., (2001) Filosofía y Poesía. Madrid, Fondo de Cultura Económica. 